\title{
Genetic determination of mortality rate in Danish dairy cows: A multivariate competing risk analysis based on the number of survived lactations
}

\author{
R. P. Maia, ${ }^{*}$ B. Ask,† P. Madsen, ${ }^{\star}$ J. Pedersen,‡ and R. Labouriau ${ }^{\star 1}$ \\ *Department of Molecular Biology and Genetics, Aarhus University, DK-8830 Tjele, Denmark \\ †Danish Pig Production, DK-1690 Copenhagen, Denmark \\ łKnowledge Centre for Agriculture, DK-8200 Aarhus, Denmark
}

\section{ABSTRACT}

Dairy cow mortality has been steadily increasing during the last 2 decades in Denmark. This study aims to verify whether genetic mechanisms might be contributing to this increase. To do so, the records of 880,480 Holstein, 142,306 Jersey, and 85,206 Red Danish dairy cows calving from 1990 to 2006 were retrieved from the Danish Cattle register. Two causes of culling of cows were considered: death and slaughtering. Bivariate competing risk genetic models with a sire model structure were used to describe the death and the slaughtering rates simultaneously. The models included 2 random components: a sire random component with pedigree representing the sire genetic effects and a herd-yearseason component. Moreover, the level of heterozygosity and the sire breed proportions were included in the models as covariates to account for potential nonadditive genetic effects due to the massive introduction of genetic material from other populations. The correlations between the sire components for death rate and slaughter rate were negative and small for the 3 populations, suggesting the existence of specific genetic mechanisms for each culling reason and common concurrent genetic mechanisms. In the Holstein population, the effects of the changes in the level of heterozygosity, breed composition, and the increasing genetic trend acted in the same direction, increasing the death rate in recent years. In the Jersey population, the effects of the level of heterozygosity and the breed proportion were small, and only the increasing genetic trend can be pointed as a genetic cause to the observed increase in the mortality rate. In the Red Danish population, neither the time-development pattern of the genetic trend nor the changes in the level of heterozygosity and breed composition could be causing the observed increase in the mortality; thus, nongenetic factors must be causing this negative development.

Key words: longevity, genetic trend, survival model

Received April 25, 2013

Accepted November 19, 2013

${ }^{1}$ Corresponding author: rodrigo.labouriau@agrsci.dk

\section{INTRODUCTION}

Dairy cow mortality has been steadily increasing during the last 2 decades all over the world. For example, the mortality rate increased from 2.6 to $5.7 \%$ in the United States from 1996 to 2007 (Garry, 2009), and in Ireland from 3.3 to $4.4 \%$ between 2002 and 2006 (Maher et al., 2008). In Denmark, cow mortality increased from an average of $2 \%$ in 1990 to approximately $3.5 \%$ in 1999 (Thomsen et al., 2004), and to $4.9 \%$ in 2005 (Thomsen and Sørensen, 2008). Cow mortality, therefore, constitutes a problem of animal welfare and farm economy. Several herd-level risk factors for mortality have been identified, such as herd size, SCC, and milk yield (Thomsen and Houe, 2006; Thomsen and Sørensen, 2009; Alvåsen et al., 2012). Moreover, concerns exist that historic breeding objectives focusing on production traits with negative genetic correlation to functional and health traits may have contributed to the observed increase in cow mortality. The goal of this work was to verify whether these concerns are well founded by studying in detail 3 populations of dairy cows, namely Holstein (HOL), Jersey (JER), and Red Danish (RDC), under production in Denmark. This task was not straightforward for 2 main reasons: the presence of incomplete observations and the presence of nonadditive genetic effects.

In the analyses presented here, we distinguish 2 causes of culling of cows: death and slaughtering. Our primary interest was to characterize the risk of dying and slaughtering will be seen here just as a competing cause of culling. The longevity of cows under production is, to the best of our knowledge, operationally defined by pooling the culling due to death and the culling due to slaughtering together. As a consequence, the large amount of evidence accumulated in the literature of the presence of genetic determination of culling rates (Ducrocq, 1994; Vollema and Groen, 1996; Caraviello et al., 2004) cannot be used to characterize the genetic determination of culling by death. In this paper, we propose a methodology that allows distinguishing these 2 causes of culling and to determine to which extent the genetic mechanisms involved with these culling causes 
overlap. We anticipated that we would show evidence of genetic determination of both causes of culling cows in the 3 populations studied; furthermore, the overlap of the genetic mechanisms involved in the 2 causes of culling would be small.

Although good-quality registers are available for the 3 Danish populations of dairy cows studied, part of the information on death culling is incompletely observed. Indeed, cows under production can leave the herd by causes different than death (e.g., export or slaughter not due to euthanasia) and, therefore, the time of death of these animals is only known to be larger than a certain observed time (i.e., we have right censuring). Moreover, some of the factors known to affect the risk of deaths vary along time and it is important to account for that when modeling these data. Finally, some animals entered the study already at an advanced age (late entry); these animals should be included in the analysis if we want to properly evaluate the number of animals at risk of dying at a certain time point. These problems can be circumvented by using statistical methods of survival analysis (Andersen et al. 1997; Kalbfleisch and Prentice, 2002; for a general overview and for applications in animal breeding see Ducrocq et al., 1988; Giolo and Demétrio, 2011). However, the survival analysis techniques currently used in animal evaluation require the censoring mechanism to be noninformative (i.e., the probability of censoring should not depend on any of the explanatory variables used in the model to describe the distribution of the time to death). Our results will show that, as one might suspect, common factors exist affecting both the time to death and the probability of a cow being slaughtered, so we clearly have informative censoring, which rules out the use of the standard techniques of survival analysis without proper adaptation. We will introduce here a statistical methodology based on multivariate competing risk models that circumvent the problem of informative censoring and still well represent the genetic scenario in a way that resembles the representation based on Gaussian linear mixed models classically used in quantitative genetics. We will simultaneously model the time-development of the probability of dying and the probability of being slaughtered (conditional on survival up to a given time) using a suitable bivariate model. This will allow us to properly characterize the quantitative genetic determination of the culling rate of cows due to death (accounting for the removal due to slaughtering) and to access the degree of overlap of the additive genetic mechanisms related to the 2 causes of culling of dairy cows in each of the 3 populations studied.

The second complication of this study was the presence of nonadditive genetic factors affecting the culling rates of dairy cows. Both the level of heterozygosity and the breed composition varied along time in our study. Moreover, the observed patterns of variation in recent years were not the same for the 3 populations studied. The models implemented here account for these genetic factors and allow us to estimate their effects. We anticipated that we would detect non-negligible effects of those factors on the culling rates associated with death (and on the culling rates due to slaughtering). Therefore, although these genetic effects are transitory (in the sense that they are not necessarily directly passed to the offspring) they should be taken into account when analyzing the time development of the mortality rate of dairy cows.

In summary, this paper aims to present a methodology to access possible genetic causes of the observed increase in mortality rate in dairy cows, which includes additive genetic effects, changes in the breed composition, and variations in the level of heterozygosity. This will be illustrated using the HOL, JER, and RDC populations of dairy cows under production in Denmark.

\section{MATERIALS AND METHODS}

\section{Data Editing}

The data used were provided by the Danish Knowledge Centre for Agriculture (Aarhus, Denmark) and contained records of all the calf births that occurred from 1990 to 2006 for the HOL, JER, and RDC dairy cattle populations. The data consist of 1 record per calving for each cow and included the following information: culling day and reason (dead or slaughtered) if the animal was culled, age at the first calving, calving year and season, herd year size (number of calf births in that particular herd year class), and coefficient of heterozygosity and sire breed composition (gene proportion of different breeds that compose the sire). The coefficient of heterozygosity and the sire breed proportions were included in the data to account for potential effects of the massive introduction of genetic material from other populations.

We considered in this study only the herds with more than 30 calves per year and that presented a stable or increasing herd size over the period in study. This data editing was done to avoid possible distortions due to the fact that the decision of culling a cow must be influenced by the herd situation. To do so, a simple linear regression of the herd size against the year was fitted and the herds with significant decreasing size, at a level of $5 \%$, were excluded from the study. Cows with age at first parity lower than $540 \mathrm{~d}$ or larger than 1,280 $\mathrm{d}$ and cows with unknown sire were eliminated. After this editing, the data set included records on 880,480 
HOL, 142,306 JER, and 85,206 RDC dairy cows. For each of the 3 breeds, a sire dam pedigree file for all sires with progenies in the edited data was extracted from the Nordic Cattle Genetic Evaluation (NAV; http:// www.nordicebv.info/Forside.htm) pedigree file.

\section{Breed Proportion and Heterosis}

The breed proportion and the coefficient of heterozygosity are currently considered in several models by the NAV. The proportion of genes from different breeds carried by each animal was calculated as follows:

$$
b_{i}(p)=\frac{b_{i}(s)+b_{i}(d)}{2},
$$

where $b_{i}(p), b_{i}(s)$, and $b_{i}(d)$ are the proportion of genes from breed $i$ in the progeny, sire, and dam, respectively. The coefficients of specific heterozygosity between the pair of breeds $i$ and $j\left[\gamma_{i j}(p)\right]$ were calculated using the method described by López-Villalobos et al. (2010), as follows:

$$
\gamma_{i j}(p)=b_{i}(s) b_{j}(d)+b_{j}(s) b_{i}(d) .
$$

The coefficient of general heterozygosity, referred to below simply as the coefficient of heterozygosity for the progeny $p$, is given by the sum of all the coefficients of specific heterozygosity for that animal.

\section{The Competing Risk Problem}

The longevity trait understood as a measure of the productive life of the cow was defined as the total number of survived lactations until the culling day. As usual in survival analyses, the data include 2 types of incomplete records: (1) right censored, comprising cows that were still alive at the end of the data collection period (January 7, 2011) or cows moved to another herd or exported during the study period and (2) late entry, comprising the cows that in the beginning of the studied period had already completed more than 1 parity (i.e., the first parity occurred before 1990). For example, if a cow had the first and the second parity before 1990 and further parities in 1990 or later, we considered only the records from the third parity and treated them as late-entry data. Although we know that this animal was alive before 1990, we were not interested in computing the risk of culling in the previous years and, therefore, we ignored the data previous to 1990 . Note that the inclusion of the animals with late-entry data in the analyses allowed us to compute the correct culling risk in each year from 1990 to 2006. Note also that no left-censored animals (i.e., animals that were culled before 1990) were included in this study because the animals culled before 1990 were not included in the data set. This does not affect the estimates of the hazard function at any time of the observation period because the left-censored animals would never be in the risk sets at any time in the observation period. The analyses concentrated on the longevity up to 6 parities; cows that completed 7 or more parities had the number of lactations truncated at the sixth parity and were treated as right censored at the sixth parity.

In this study, we dealt with a competing risk problem, as the cows may have been culled for 1 of 2 possible reasons (Martinussen and Scheike, 2006), namely death or slaughter. A cow was registered as dead when the animals suddenly died or when the animals were euthanized. To describe the competing risk models considered here, we define the random variables $T$, representing the number of survived lactations and including values in $\{1,2,3,4,5,6\}$, and $J$, representing the culling cause. The cause-specific hazard probability (Kalbfleisch and Prentice, 2002) for the jth culling cause is given, for $t=1, \ldots, 6$, by

$$
\lambda_{j}(t)=P(T=t, J=j \mid T \geq t) .
$$

Here, $j$ is equal to 1 if the cow died and 2 if the cow was slaughtered. That is, $\lambda_{j}(t)$ is the probability of a cow being culled for the $j$ th specific cause at the th lactation, given that the cow had survived from all causes until the th lactation. The survival function is then defined by

$$
S(t)=P(T>t)=\prod_{k \leq t}[1-\lambda \cdot(k)],
$$

where $\lambda .(t)=\lambda_{1}(t)+\lambda_{2}(t)$ is the total hazard probability of being culled at the th lactation.

\section{Multivariate Mixed Models}

We handled the competing risk problem by using a bivariate discrete relative risk model with frailty components (BFDRRM). In the following description of the BFDRRM, $\mathbf{U}=\left(\mathbf{U}_{1}, \mathbf{U}_{2}\right)$ is a set of random components (i.e., frailties as referred to in the literature of survival analysis), assuming different effects for each specific cause, $X(t)$ represents a set of explanatory variables containing time-dependent and time-independent explanatory variables, and $Z$ represents a set of explanatory variables defining the random effects. According to the BFDRRM, the vector of the specific hazard probability functions for the $i$ th cow is given, conditionally on $\mathbf{U}=\mathbf{u}$, by 
$\left[\begin{array}{c}\lambda_{i 1}(t \mid \mathbf{u}) \\ \lambda_{i 2}(t \mid \mathbf{u})\end{array}\right]=\left[\begin{array}{c}\lambda_{01}(t) \exp \left(X_{i}^{\prime}(t) \boldsymbol{\beta}_{1}+Z_{i}^{\prime} \mathbf{u}_{1}\right) \\ \lambda_{02}(t) \exp \left(X_{i}^{\prime}(t) \boldsymbol{\beta}_{2}+Z_{i}^{\prime} \mathbf{u}_{2}\right)\end{array}\right]$ for $t=1,2, \ldots, 6$,

where $\lambda_{0 j}(t)$ is the baseline hazard function for the specific-cause $j$ at lactation $t, \boldsymbol{\beta}_{1}$ and $\boldsymbol{\beta}_{2}$ are vectors of parameters representing the fixed effects for the specific cause 1 or 2 , respectively, and $\lambda_{i j}(t \mid \mathbf{u})$ is the short notation for $\lambda_{i j}(t \mid \mathbf{U}=\mathbf{u})$. The time-independent explanatory variables included in the model were age at first parity (first quartile, second-to-third quartile, and fourth quartile), coefficient of heterozygosity, and the sire breed proportion (proportion of Holstein-Friesian genes for HOL sires, US Jersey genes for JER sires, and Holstein-Friesian, American Brown, and Nordic Red genes for the RDC sires). The following time-dependent explanatory variables were included in the model: the herd size given by the total number of observed calf births per year (first quartile, second-to-third quartile, and fourth quartile), the calving year, and the calving season (first semester aggregating the winter and spring, and second semester aggregating summer and fall). We included in the model 2 random components (i.e., frailties): a sire random component $\mathbf{S}$, with pedigree representing the sire genetic effect and a herdyear-season component $\mathbf{H}$. We assumed that $(\mathbf{S}, \mathbf{H})^{\prime}$ followed a multivariate normal distribution, with mean equal to zero and covariance matrix given by

$$
\boldsymbol{\Sigma}=\left[\begin{array}{cc}
\boldsymbol{\Sigma}_{s} \otimes \mathbf{A} & 0 \\
0 & \boldsymbol{\Sigma}_{h} \otimes \mathbf{I}
\end{array}\right] .
$$

Here, $\boldsymbol{\Sigma}_{s}=\left[\begin{array}{cc}\sigma_{s 1}^{2} & \sigma_{s 12} \\ \sigma_{s 12} & \sigma_{s 2}^{2}\end{array}\right]$ represents the covariance matrix for the sire effect $\left(\sigma_{s 1}^{2}\right.$ and $\sigma_{s 2}^{2}$ are the genetic variances for cause 1 and 2, respectively; that is, the variances of $\mathrm{S}_{1}$ and $\mathrm{S}_{2}$; and $\sigma_{s 12}$ is the genetic covariance; that is, the covariance between $S_{1}$ and $S_{2}$ ), $A$ is a known relationship matrix given by sire pedigree, $\boldsymbol{\Sigma}_{h}=\left[\begin{array}{cc}\sigma_{h 1}^{2} & \sigma_{h 12} \\ \sigma_{h 12} & \sigma_{h 2}^{2}\end{array}\right]$ represents the covariance matrix for the herd-year-season effect $\left(\sigma_{h 1}^{2}\right.$ and $\sigma_{h 2}^{2}$ are the herd-year-season random components related to the specific cause 1 and 2, respectively, and $\sigma_{h 12}$ is the covariance between the herdyear-season random components), and $\mathbf{I}$ is an identity matrix.

\section{Inference}

The bivariate discrete competing risk model may be seen as a sequence of multinomial trials (Kalbfleisch and Prentice, 2002), where each of the lactations is considered as a new trial and can be modeled via a multivariate binomial model with random components. To do so, we created a pseudo data set containing 1 record for each of the observed lactations of each cow. Next, we created 2 indicator variables $\left(Y_{i t 1}\right.$ and $\left.Y_{i t 2}\right)$, where $Y_{i t j}$ receives the value 1 if the cow was culled by the reason $j$ at lactation $t$ and 0 otherwise. Then, we assumed that $Y_{i t} \mid \mathbf{U}=\mathbf{u}$ were pseudo Bernoulli variables with probability of success equal to $\lambda_{j}(t \mid \mathbf{U}=\mathbf{u})$ and that $\operatorname{Cov}\left(Y_{i t 1}, Y_{i t 2} \mid \mathbf{U}=\mathbf{u}\right)=0$. Maia et al. (2013) showed that the distribution of the pseudo binomial variables could be approximated by a Poisson distribution as a result of the classical approximation of the binomial distribution by the Poisson distribution applied when the probability of success is very small (i.e., the law of rare events). The use of this approximation avoided serious numerical and statistical instability, as the present problem would be equivalent to perform inference with a Bernoulli model with the probability of success very close to zero. The model also included a dispersion parameter via quasi-likelihood estimation (Wedderburn, 1974; Breslow and Clayton, 1993; Jørgensen and Labouriau, 2012; Jørgensen et al., 1996). In this approach, it is assumed that $\operatorname{Var}\left(\mathrm{Y}_{\mathrm{it} j} \mid \mathbf{U}=\mathbf{u}\right)$ $=\phi_{j} \lambda_{j}(t \mid \mathbf{U}=\mathbf{u})$, where $\phi_{j}$ represents the parameter of dispersion for the $j$ th specific cause. All the models were fitted using the software DMU version 6.0, release 5.1 (Madsen and Jensen, 2010; Madsen et al. 2010).

\section{Heritability, Breeding Values, and Genetic Trend}

The marginal heritability for the relative risk of culling by each specific cause $j$ at the lactation $t$ is calculated as follows:

$$
h_{\lambda(t)}^{2} \approx \frac{\sigma_{s, j}^{2}}{\sigma_{s, j}^{2}+\sigma_{h, j}^{2}+\frac{\phi_{j}}{\lambda_{j}^{*}(t)}}
$$

where $\phi_{j}$ represents the dispersion parameter and $\lambda_{j}^{*}(t)$ represents the hazard probability for the $j$ th cause evaluated at the predicted values of the random components. Equation [1] arises from a Taylor approximation of the total variance and the theory of survival analysis [for details, see Maia et al. (2013)]. More precisely, define, for each lactation $t=1,2, \ldots, 6$, for each individual $i=1, \ldots, n_{t}$ at risk at lactation $t$ and the $j$ th cause of culling $(j=1,2)$,

$$
\hat{\eta}_{i, j}(t)=\widehat{\log \left[\lambda_{0 j}(t)\right]}+\mathbf{X}_{i}^{t}(t) \hat{\boldsymbol{\beta}}_{j}+\mathbf{Z}_{1 i}^{t} \widehat{\mathbf{s}_{j}}+\mathbf{Z}_{2 i}^{t} \widehat{\mathbf{h}_{j}}
$$


Table 1. Some survival statistics

\begin{tabular}{lccc}
\hline & \multicolumn{3}{c}{ Dairy cow breed } \\
\cline { 2 - 4 } Item & Holstein & Jersey & Danish Red \\
\hline Median survival time (no. of lactations) & 2 & 2 & 3 \\
Probability of survival 7 or more parities & 0.031 & 0.011 & 0.007 \\
Proportion of censoring (\%) & 19.9 & 22.7 & 15.6 \\
\hline
\end{tabular}

as the estimated of the cause-specific log hazard probability (the linear predictor) and the $\lambda_{j}^{*}(t)=\exp \left[\hat{\eta}_{i, j}(t)\right]$. The heritability was estimated at the overall median survival time $t_{m}$ and the specific hazard probability for the specific cause $j$ at the median survival time was estimated by

$$
\tilde{\lambda}_{j}\left(t_{m}\right)=\exp \left[\bar{\eta}_{j}\left(t_{m}\right)\right]
$$

where $\bar{\eta}_{j}\left(t_{m}\right)=n_{t_{m}}^{-1} \sum_{i=1}^{n_{t_{m}}} \hat{\eta}_{i, j}\left(t_{m}\right)$.

The genetic trend for each specific cause of culling was estimated by the mean of the sire EBV by the birth year. Only EBV of young bulls were considered to avoid the effect of very high selected sires. A bull for which the difference between its birth year and the birth year of its oldest daughter presented in the data is smaller or equal to 5 yr was declared to be a young bull.

\section{RESULTS AND DISCUSSION}

The evolution of the mortality rate in the edited data set is displayed in Figure 1, where an almost linear increase is observed over the studied period for the 3 populations. This is in agreement with the increase in the mortality of cows in Denmark reported in Thomsen and Sørensen (2008) and is in line with the results obtained in other countries (e.g., Maher et al., 2008; Garry, 2009).

The median survival times were 2 lactations for the RDC and the HOL populations and 3 lactations for the JER population. The proportions of censored data were relatively high ( $\sim 15-22 \%$; see Table 1$)$ and the probability of a cow to survive 7 or more lactations were low (less than 3\%; see Table 1). Figure 2 presents the proportions of dead, slaughtered, and censored cows for each of the parities.

Applying the specially designed multivariate extension of the BFDRRM for competing risks described below allowed us to simultaneously model the culling rate of cows related to death and the culling rate due to slaughtering. The estimates of the sire variances for slaughtering were almost equal for the 3 populations $(\sim 0.02$, with $\mathrm{SE} \sim 0.001)$ and the marginal heritabilities for slaughtering (evaluated at the median survival probability) varied from approximately $3 \%$ to approximately $4 \%$, being slightly larger for the RDC population (see Table 2). On the other hand, the estimates of the sire variances for death varied considerably (largest for the RDC and smallest for JER). Moreover, the marginal heritabilities for death were smaller than the heritabilities for slaughter for the HOL and JER populations and were larger than the heritabilities for slaughter for the RDC population (see Table 2). Furthermore, the correlations between the sire random components for death and the sire random component for slaughtering were negative but small for the $3 \mathrm{popu}-$ lations (although not statistically significant for the JER population; see Table 2), suggesting the existence of common concurrent genetic mechanisms related to the slaughtering and the death rates for the HOL and the RDC populations. On the other hand, because the absolute values of those genetic correlations are small, specific genetic mechanisms must also exist for each of the culling reasons.

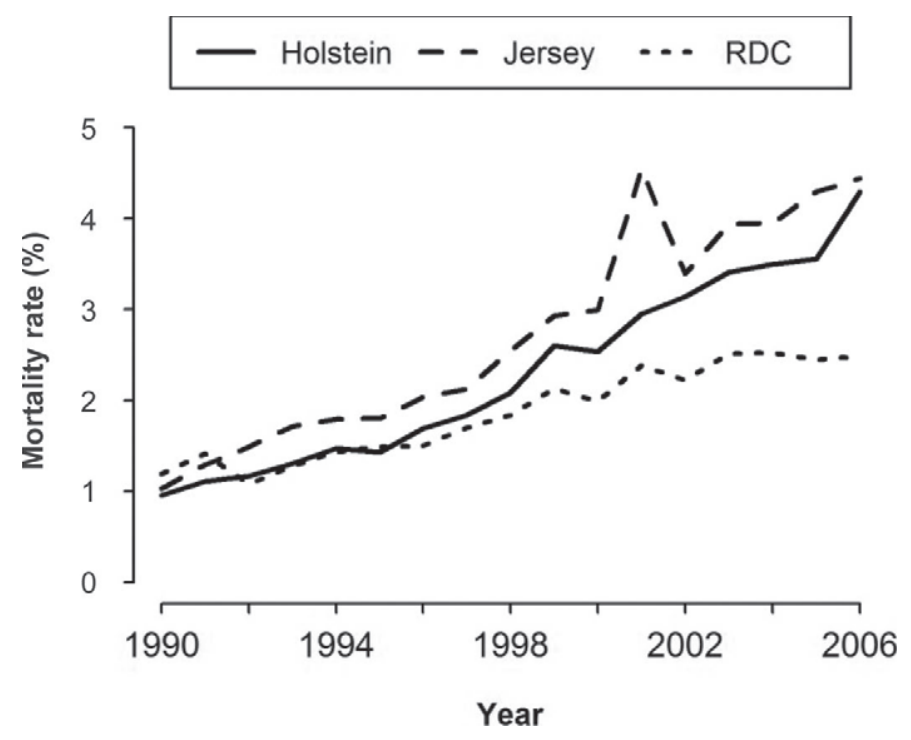

Figure 1. Mortality rate evolution from 1990 to 2006. RDC $=$ Red Danish dairy cows. 

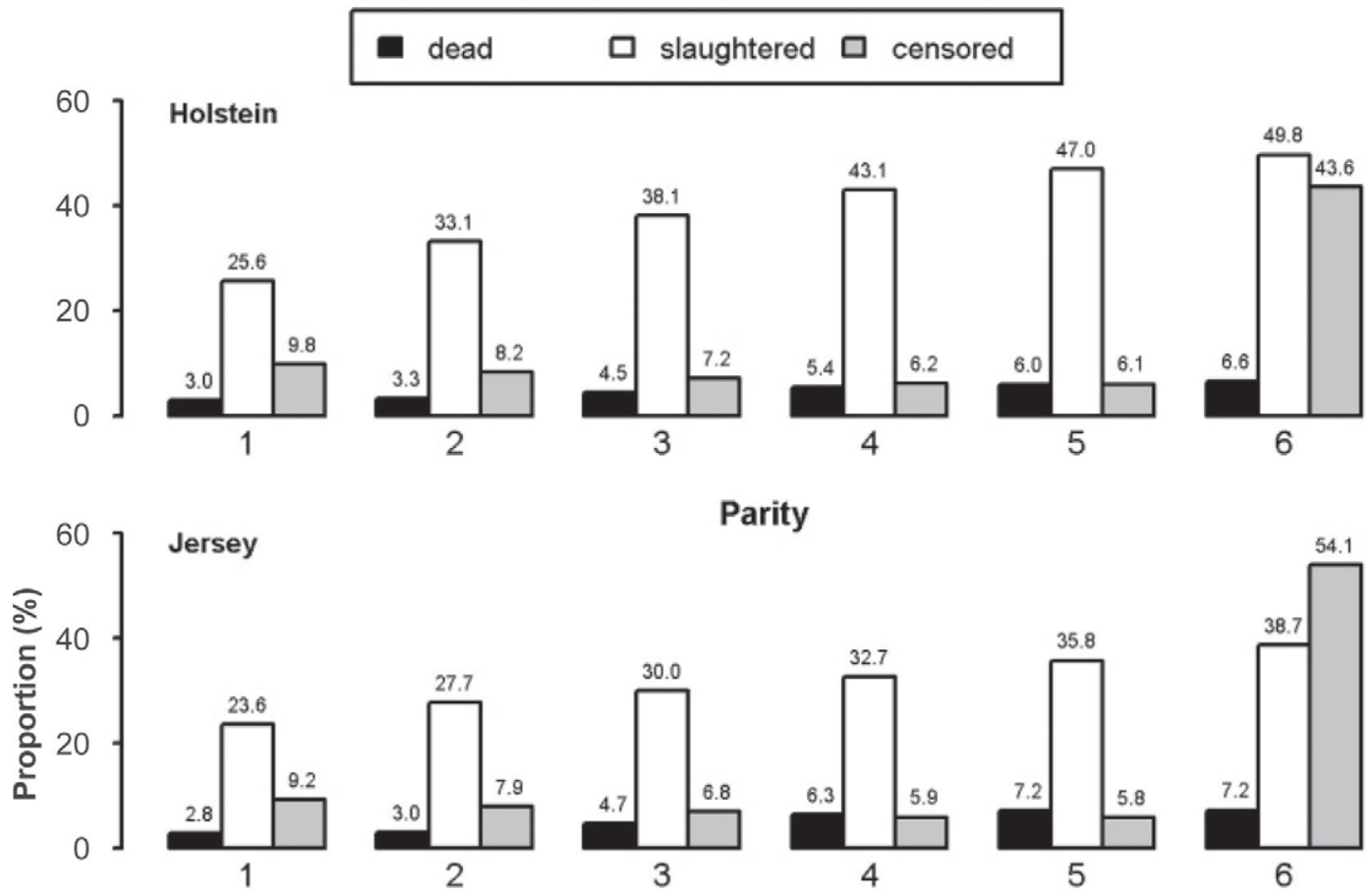

Parity
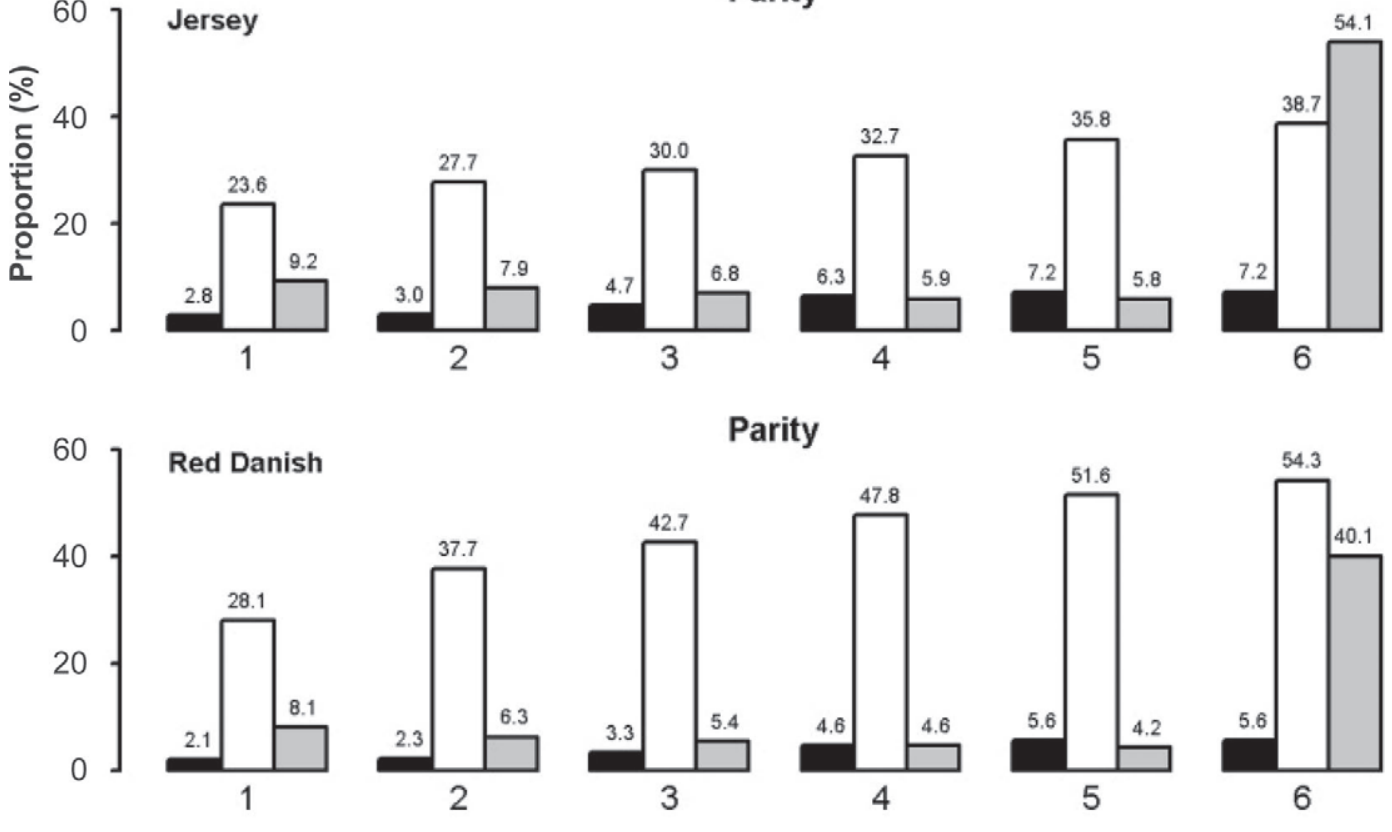

Parity

\section{Parity}

Figure 2. Proportion of dead, slaughtered, and censored cows per parity.

The effects of the coefficient of heterozygosity on the hazard probability for death were all negative (see Table 3). However, the recent time developments of the level of heterozygosity were not the same for the 3 populations (see Figure 3); therefore, the effects of changes in the coefficient of heterozygosity were dif-

Table 2. Estimates of the variance and correlations of random components from the competing risk model and the corresponding marginal heritabilities

\begin{tabular}{|c|c|c|c|c|c|c|c|}
\hline Component $^{1}$ & Cause & \multicolumn{2}{|c|}{ Holstein } & \multicolumn{2}{|c|}{ Jersey } & \multicolumn{2}{|c|}{ Red Danish } \\
\hline \multirow[t]{2}{*}{ S } & Death & $0.155(0.007)$ & \multirow[t]{2}{*}{$-0.079(0.031)$} & $0.089(0.013)$ & \multirow[t]{2}{*}{$-0.094(0.084)$} & $0.436(0.040)$ & \multirow[t]{2}{*}{$-0.20(0.072)$} \\
\hline & Slaughter & $0.022(0.001)$ & & $0.022(0.002)$ & & $0.020(0.002)$ & \\
\hline \multirow[t]{2}{*}{$\mathrm{H}$} & Death & $0.458(0.006)$ & \multirow[t]{2}{*}{$-0.26(0.010)$} & $0.414(0.014)$ & \multirow[t]{2}{*}{$-0.37(0.025)$} & $0.955(0.031)$ & \multirow[t]{2}{*}{$-0.46(0.032)$} \\
\hline & Slaughter & $0.048(0.001)$ & & $0.053(0.002)$ & & $0.030(0.002)$ & \\
\hline Dispersion & Death & $0.683(0.001)$ & - & $0.712(0.002)$ & - & $0.514(0.002)$ & - \\
\hline Heritability & Slaughter & \multicolumn{2}{|c|}{0.043} & \multicolumn{2}{|c|}{0.036} & \multicolumn{2}{|c|}{0.047} \\
\hline
\end{tabular}

${ }^{1} \mathrm{~S}$ represents the sire random component and $\mathrm{H}$ represents the herd-year-season random component. 
Table 3. Estimated mean effect of the coefficient of heterozygosity on the longevity for each culling reason (asymptotic SE in parentheses)

\begin{tabular}{lcr}
\hline Population & Death & \multicolumn{1}{c}{ Slaughter } \\
\hline Holstein & $-0.212(0.036)$ & $0.032(0.011)$ \\
Jersey & $-0.013(0.067)$ & $-0.127(0.025)$ \\
Red Danish & $-0.266(0.116)$ & $-0.299(0.033)$ \\
\hline
\end{tabular}

ferent in the 3 populations. For instance, in the HOL population, the level of heterozygosity decreased along time, which contributed to generate a concomitant increase in the mortality rate of this population along the years. In contrast, in the RDC population, the pronounced increase observed in the level of heterozygosity along the years acted to decrease the mortality. In the JER population, although the level of heterozygosity increased along the years, the effect of the coefficient of heterozygosity on the hazard probability of death was small (and even not statistically significantly different from zero); therefore, the positive influence of the increase in the level of heterozygosity on the mortality (if any) must also have been small. Furthermore, the effects of the historical changes on the breed composition on the hazard probability for death resemble the effects of changes in level of heterozygosity discussed above. Indeed, for the HOL population, the proportion of Holstein-Friesians had a positive effect on the hazard probability for death (see Table 4), which tended to increase the hazard probability for death for this population along the years because the proportion of Holstein-Friesian increased in the period of study (see Figure 4). The effects of changes in breed composition

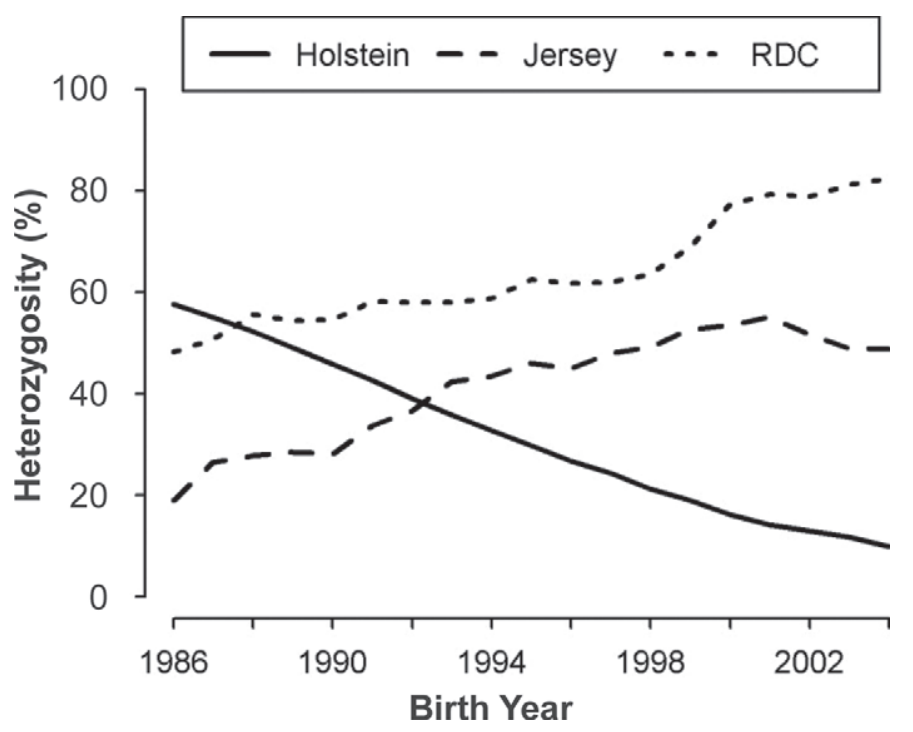

Figure 3. Average coefficient of heterozygosity of the cows by birth year. $\mathrm{RDC}=$ Red Danish dairy cows.
Table 4. Estimated mean effects of the sire breed compositions on each specific culling rate (asymptotic SE in parentheses)

\begin{tabular}{llcc}
\hline Population & Breed & Death & Slaughter \\
\hline Holstein & Holstein-Friesian & $0.487(0.107)$ & $-0.095(0.033)$ \\
\multirow{2}{*}{ Jersey } & US Jersey & $0.146(0.128)$ & $-0.016(0.057)$ \\
\multirow{2}{*}{ Red Danish } & Holstein-Friesian & $0.303(0.261)$ & $-0.169(0.061)$ \\
& American Brown & $0.053(0.218)$ & $-0.003(0.049)$ \\
& Nordic & $0.208(0.243)$ & $-0.167(0.599)$ \\
\hline
\end{tabular}

for the JER and the RDC populations tended to be small and were not statistically different from zero (see Table 4).

The observed marginal genetic trends differed substantially for the slaughter- and death-related components of the longevity (see Figure 5 and Table 5). For slaughter, all 3 populations showed a decreasing average sire genetic effect on the hazard probability, suggesting that the recent selection programs contributed to improvement of this component of the longevity. On the other hand, the average sire genetic effect on the hazard probability to death showed a clear increasing trend for the HOL and the JER populations, suggesting that the recent selection programs contributed to increase the mortality rate in those populations. Furthermore, the genetic trend for death was almost constant for the RDC population, with a small decrease in the last years, suggesting that the recent selection programs were essentially acting neutrally on the death-related longevity for this population. Furthermore, the fact that qualitative differences existed in the genetic trends estimated for slaughter and death corroborates to the hypothesized existence of different specific determining genetic mechanisms related to the 2 culling causes in discussion.

In summary, in the HOL population, the effects of the changes in the level of heterozygosity and breed composition acted in the same direction as the increasing genetic trend for death; therefore, all these effects tended to increase the mortality rate for the HOL population along the years. The case of the JER population occupied an intermediary position. In this population,

Table 5. Spearman correlation between the EBV and the sire birth year

\begin{tabular}{llcc}
\hline Population & Culling & $\begin{array}{c}\text { Spearman } \\
\text { correlation }\end{array}$ & $P$-value \\
\hline Holstein & cause & 0.25 & $<0.001$ \\
\multirow{2}{*}{ Jersey } & Death & -0.44 & $<0.001$ \\
& Slaughter & 0.24 & $<0.001$ \\
Red Danish & Death & -0.41 & $<0.001$ \\
& Slaughter & -0.03 & 0.28 \\
& Death & -0.53 & $<0.001$ \\
\hline
\end{tabular}



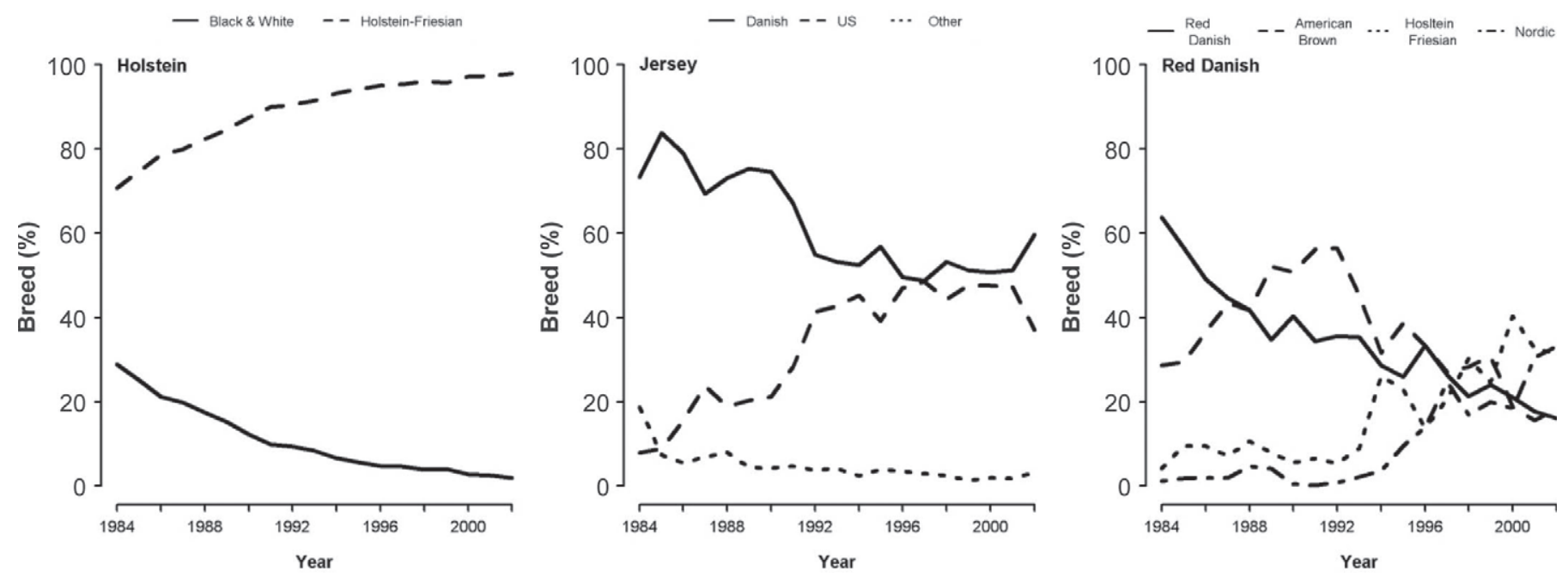

Figure 4. Average breed composition of the sires by the birth year. US = United States.

the effects of the changes in the level of heterozygosity and breed composition were positive and small (if any), and only the increasing genetic trend could be pointed to as a genetic cause of the observed increase in the mortality rate. In contrast, in the RDC population, neither the time-development pattern of the genetic trend nor the changes in the level of heterozygosity and breed composition could have caused the observed increase in the mortality rate. This suggests that strong nongenetic causes of mortality of cows must have been present in the RDC population.

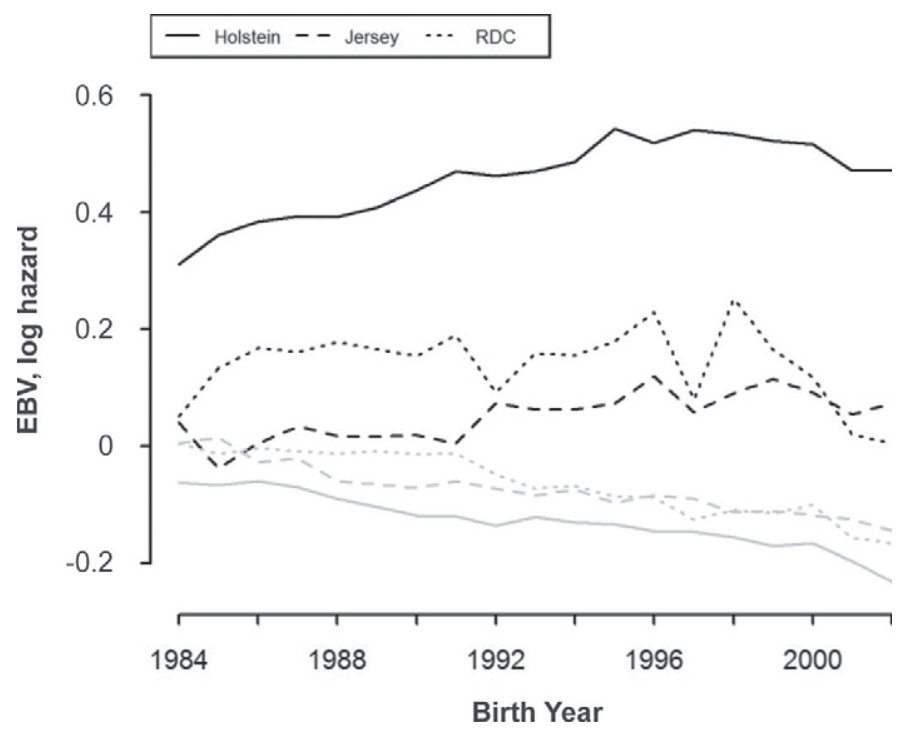

Figure 5. Marginal genetic trends for the sire EBV for death (black curves) and slaughter (gray curves). RDC $=$ Red Danish dairy cows.

\section{CONCLUSIONS}

Our results indicate that the increase in cow mortality that has occurred recently in Denmark can be partially explained by genetic causes. Although evidence exists that genetic-related mechanisms are associated with the increase in mortality of cows for the HOL and the JER populations, no evidence was found of such deleterious genetic effects in the RDC population. As the mortality rate of the $\mathrm{RDC}$ is also increasing, nongenetic factors must be causing this negative development. Furthermore, multivariate methods using competing risk methods are required for properly discussing the type of questions we answered here because evidence exists of the presence of different specific genetic mechanisms related to culling due to slaughter and culling due to death. The multivariate discrete relative risk model for competing risks presented here is an example of such a technique. It would be interesting to perform investigations similar to the present study in other populations; the statistical tools for that are available now.

\section{ACKNOWLEDGMENTS}

R. P. Maia was financed by the project "Svineavl: Developing New Methods for Genetic Selection of Sow Durability" (Ministry of Food, Agriculture and Fisheries, Copenhagen, Denmark).

\section{REFERENCES}

Alvåsen, K., M. Jansson Mörk, C. Hallén Sandgren, P. T. Thomsen and U. Emanuelson. 2012. Herd-level risk factors associated with cow mortality in Swedish dairy herds. J. Dairy Sci. 95:4352-4362. Andersen, P. K., Ø. Borgan, R. D. Gill, and N. Keiding. 1997. Statistical Models Based on Counting Processes. Springer-Verlag, New York, NY. 
Breslow, N. E., and D. G. Clayton. 1993. Approximate inference in generalized linear mixed models. J. Am. Stat. Assoc. 88:9-25.

Caraviello, D. Z., K. A. Weigel, and D. Gianola. 2004. Prediction of longevity breeding values for US Holstein sires using survival analysis methodology. J. Dairy Sci. 87:3518-3525.

Ducrocq, V. 1994. Statistical analysis of length of productive life for dairy cows of the Normande breed. J. Dairy Sci. 77:855-866.

Ducrocq, V., R. L. Quaas, E. J. Pollak, and G. Casella. 1988. Length of productive life of dairy cows. 1. Justification of a Weibull model. J. Dairy Sci. 71:3061-3070.

Garry, F. 2009. How big of a problem is adult cow mortality? Western Dairy News 9:W-31-W-32.

Giolo, S. R., and C. G. B. Demétrio. 2011. A frailty modeling approach for parental effects in animal breeding. J. Appl. Stat. 38:619-629.

Jørgensen, B., and R. Labouriau. 2012. Exponential Families and Theoretical Inference. Vol. 52. 2nd ed. Monografías de Matemática, Rio de Janeiro, Brazil.

Jørgensen, B., R. Labouriau, and S. Lundbye-Christensen. 1996. Linear growth curve analysis based on exponential dispersion models. J. R. Stat. Soc. B 58:573-592.

Kalbfleisch, J. D., and R. L. Prentice. 2002. The Statistical Analyses of Failure Time Data. 2nd ed. Wiley Series in Probability and Statistics, John Wiley \& Sons Inc., Hoboken, NJ.

López-Villalobos, N., M. Penasa, R. D. Zotto, M. Cassandro, W. Brade, O. Distl, R. Evans, and A. Cromie. 2010. Calculation of a cow culling merit index including specific heterosis in a multibreed dairy population. Arch. Tierzucht 53:9-17.

Madsen, P., and J. Jensen. 2010. A User's Guide to DMU: A Package for Analysing Multivariate Mixed Models. Version 6, release 5.0. Aarhus University, Faculty of Agricultural Sciences, Tjele, Denmark.
Madsen, P., G. Su, R. Labouriau, and O. F. Christensen. 2010 DMU-A package for analyzing multivariate mixed models. Page 732 in Proc. 9th World Congress on Genetics Applied to Livestock Production (WCGALP), Leipzig, Germany. Gesellschaft für Tierzuchwissenschaften e. V., Neustadt, Germany.

Maher, P., M. Good, and S. J. More. 2008. Trends in cow numbers and culling rate in the Irish cattle population, 2003 to 2006 . Ir Vet. J. $61: 455-463$.

Maia, R. P., P. Madsen, and R. Labouriau. 2013. Multivariate survival mixed models for genetic analysis of longevity traits. J. Appl. Stat. http://dx.doi.org/10.1080/02664763.2013.868416.

Martinussen, T., and T. H. Scheike. 2006. Dynamic Regression Models for Survival Data. Statistics for Biology and Health. Springer, New York, NY.

Thomsen, P. T., and H. Houe. 2006. Dairy cow mortality. A review. Vet. Q. 28:122-129.

Thomsen, P. T., A. M. Kjeldsen, J. T. Sørensen, and H. Houe. 2004. Mortality (including euthanasia) among Danish dairy cows (19902001). Prev. Vet. Med. 62:19-33.

Thomsen, P. T. and J. T. Sørensen. 2008. Euthanasia of Danish dairy cows evaluated in two questionnaire surveys. Acta Vet. Scand. 50:33-37.

Thomsen, P. T., and J. T. Sørensen. 2009. Factors affecting the risk of euthanasia for cows in Danish dairy herds. Vet. Rec. 165:43-45.

Vollema, A. R., and A. F. Groen. 1996. Genetic parameters of longevity traits of an upgrading population of dairy cattle. J. Dairy Sci. 79:2261-2267.

Wedderburn, R. W. M. 1974. Quasi-likelihood functions, generalized linear models, and the Gauss-Newton method. Biometrika $61: 439-447$. 